\title{
Sense of Coherence in People with and without Inflammatory Bowel Diseases - Is there a Difference?
}

\author{
Timna Naftali $^{1}$, Adi Eindor-Abarbanel ${ }^{2}$, Nahum Ruhimovich ${ }^{1}$, Ariella Bar-Gil Shitrit ${ }^{3}$, Fabiana Sklerovsky-Benjaminov ${ }^{1}$, \\ Ido Laish $^{1}$, Shay Matalon ${ }^{2}$, Haim Shirin ${ }^{2}$, Yael Milgrom ${ }^{3}$, Tomer Ziv-Baran ${ }^{4}$, Efrat Broide ${ }^{2}$
}

1) Department of

Gastroenterology and

Hepatology, Meir Medical Center, affiliated with the Sackler School of Medicine, Tel Aviv University, Tel Aviv 2) The Kamila Gonczarowski Institute for Gastroenterology and Liver Diseases, Assaf Harofeh Medical Center, affiliated with the Sackler School of Medicine, Tel Aviv University, Tel Aviv

3) Digestive Diseases Institute, Shaare Zedek Medical Center, Jerusalem

4) Department of Epidemiology and Preventive Medicine, School of Public Health, Sackler Faculty of Medicine, Tel Aviv University, Tel Aviv, Israel

Address for correspondence: Adi Eindor-Abarbanel, MD Assaf Harofeh Medical Center Zrifin, 70300 Israel adiabarbanel@gmail.com

Received: 19.11.2019 Accepted: 04.02.2019

\section{ABSTRACT}

Background \& Aims: Sense of coherence (SOC) is a theoretical concept reflecting a persons resources and orientation, which enable individuals to cope with stressors in a health-promoting manner. In several multifactorial chronic diseases, such as diabetes mellitus and systemic lupus erythematosus, SOC was correlated with disease development. It was also related with the emotional distress in patients with inflammatory bowel disease (IBD).The aim of this study was to investigate the possible correlation between low SOC scores and the presence of IBD.

Methods: A total of 183 Crohn's disease (CD) and 71 ulcerative colitis (UC) patients completed questionnaires including demographic data and the 13 items for the SOC questionnaire. The IBD patients were matched to 124 healthy people according to age, gender, education, employment status and marital status.

Results: In the CD cohort, 96 patients were matched to the healthy cohort according to the propensity score value, and in the UC cohort 57 were matched. Patients with Crohns' disease had a median SOC score of 63 (IQR 56-71), and healthy matching controls of 62.5 (IQR 55.25- 68.75) $\mathrm{p}=0.369$. Patients with ulcerative patients had a median SOC score of 66 (IQR 56-73) and healthy controls 62 (IQR 55-69) p=0.354.

Conclusions: In our study SOC was not related to the development of IBD. The question of whether SOC is associated with the development of chronic disease and particularly with IBD remains open.

Key words: inflammatory bowel diseases - sense of coherence - Crohns disease - ulcerative colitis.

Abbreviations: CD: Crohns' disease; IBD: inflammatory bowel disease; SLE: systemic lupus erythematous; SOC: sense of coherence; UC: ulcerative colitis.

\section{INTRODUCTION}

The inflammatory bowel diseases (IBDs), consisting of ulcerative colitis (UC), Crohn's disease $(\mathrm{CD})$ and undetermined colitis, are characterized by chronic inflammation of the gastrointestinal tract in genetically susceptible individuals exposed to environmental risk factors [1].

Similar to other chronic diseases, IBDs are long-term medical conditions that influence individual's wellbeing and quality of life [2]. The pathogenesis behind the development of chronic diseases is multifactorial, and includes genetic, environmental and emotional components. Several common chronic diseases, such as diabetes mellitus [3-5] and IBD $[6,7]$ have higher rates of concurrent psychopathologies. Furthermore, patients suffering from psychological distress are less adherent to recommendations for behavioral and lifestyle modifications and therefore are prone to more severe disease, with poorer control [8].

Antonovsky, a medical sociologist, in his theory about salutogenesis, introduced the concept of Sense of Coherence (SOC) $[9,10]$. This concept is a theoretical construct that was developed to explain why some people fall ill and others do not, regardless of stressful events [11]. The theory focuses on personal resources needed to maintain health. Sense of coherence consists of three core elements: comprehensibility, manageability and meaningfulness. Comprehensibility means that the individual considers structured environmental demands. Manageability refers to the resources that an individual has to meet these demands. Meaningfulness is the understanding that these demands are worth the resources invested to deal with them. These three components are 
important parts of an individual's coping strategy. The stronger one' s SOC, the more likely a person is able to adopt appropriate coping strategies. Therefore, SOC is considered a key tool in enabling a person to deal with a stressor. According to Antonovsky's theory, SOC is stable and enduring and develops mainly during the first three decades of life [8-12].

In several multifactorial, chronic diseases, such as diabetes mellitus [13] and systemic lupus erythematosus (SLE) [14], SOC was shown to be correlated with disease development. Feritas et al. [15] conducted a study among patients with IBD and investigated the relationship between SOC, anxiety and depression. They found that lower SOC scores were independently associated with higher levels of depression and anxiety. Sense of coherence is strongly related to perceived health. In patients with diabetes mellitus [13] and SLE [14], low SOC scores were associated with the development of the disease. However, no similar studies have been conducted in IBD patients.

The literature data showed that lower SOC scores were correlated with emotional distress in IBD patients [15]. Therefore, we aimed to explore a possible association between a low SOC score and an individual's tendency to develop IBD.

\section{MATERIAL AND METHODS}

From November 2015 to May 2017, ambulatory patients at least 18 year-old, with an established diagnosis of IBD were invited to participate in this study. Patients were recruited from three university hospitals in Israel: Assaf Harofeh, Meir Medical Center and Shaare Zedek Medical Center.

All patients completed questionnaires including demographic data and the 13-item SOC questionnaire. Healthy controls were recruited online, via social media advertisements. The healthy controls answered an anonymous electronic questionnaire that included demographic data and the SOC questionnaire. Only those who declared not using any chronic medications as well as not having a chronic disease were included. Healthy controls were matched to IBD patients according to five criteria: age, gender, education, employment status and marital status. The Ethics Committees of each hospital approved the study. All patients signed the informed consent.

Sense of coherence was assessed according to the SOC orientation to life score $[16,17]$. The SOC-13 scale measures the degree to which an individual views the world as comprehensible (5 items), manageable (4 items), and meaningful (4 items) using a 7-point Likert scale. The total SOC-13 score is the sum of the items, ranging from 13 to 91 , with higher scores reflecting better SOC. The SOC 13 was validated in IBD patients [17]

\section{Statistical analysis}

Categorical variables were described using frequency and percentage. Continuous variables were evaluated for normal distribution using histograms and Q-Q plots. Normally distributed continuous variables were described as mean and standard deviation and non-normally distributed data were expressed as median and interquartile range. Kruskal-Wallis test analysis of variances (ANOVA) and Mann-Whitney test were used to compare continuous variables between categories. Categorical variables were compared using the chi-square or Fisher's exact test respectively. The correlation between continuous variables was evaluated using Spearman's rank correlation coefficient test.

Propensity score was calculated using multivariate logistic regression: age, gender, education, employment status and marital status were included in the regression. Patients in the $\mathrm{CD}$ cohort were matched to the healthy cohort according to the propensity score value. An absolute difference of up to 5\% was considered as acceptable for matching. Categorical variables were compared between the matched participants using the McNemar test and continuous variables were compared using the Wilcoxon Signed- Ranks test or the paired sample test. We repeated the same steps with the UC cohort. All statistical tests were two tailed and $\mathrm{p}<0.05$ was considered statistically significant.

The statistical analyses were performed using SPSS (IBM Corp. Released 2015. IBM SPSS Statistics for Windows, Version 24.0. Armonk, NY: IBM Corp.).

\section{RESULTS}

Among 508 adults who completed the questionnaire, 254 were ambulatory patients with consecutive clinic appointments (183 with CD and 71 with UC), whereas 124 healthy adult controls were contacted through social media and volunteered to answer the online version. In the CD cohort, 96 patients were matched to the healthy cohort according to the propensity score value, and in the UC cohort, 57 were matched. Demographic data of the two cohorts according to the matching are included in Tables I and II, respectively. Crohn's disease patients had a median SOC score of 63 (IQR 56-71) and healthy matched controls had a median score of 62.5 (IQR 55.25- 68.75). Ulcerative colitis patients had a median SOC score of 66 (IQR 56-73) and healthy controls 62 (IQR 55-69). There were no statistically significant differences between the groups ( $\mathrm{p}=0.369$ and $\mathrm{p}=0.354$, respectively).

Table I. 13-item SOC median scores in patients with Crohn's disease compared with healthy controls and demographic parameters after matching

\begin{tabular}{|c|c|c|c|}
\hline Variable & Controls & $\begin{array}{l}\text { Crohn's } \\
\text { Disease }\end{array}$ & \\
\hline Women n (\%) & $64(66.7)$ & $62(64.6)$ & \\
\hline Education $>12$ years, $\mathrm{n}(\%)$ & $79(83.3)$ & $79(83.3)$ & \\
\hline \multicolumn{4}{|l|}{ Marital Status, n (\%) } \\
\hline Single & $15(15.6)$ & $18(18.8)$ & \\
\hline In a relationship & $74(77.1)$ & $71(74)$ & \\
\hline Past relationship & $7(7.3)$ & $7(7.3)$ & \\
\hline Employed, n (\%) & $83(86.5)$ & $82(85.4)$ & \\
\hline Age median (IQR) years & $\begin{array}{c}36.71 \\
(30.55-46.01)\end{array}$ & $\begin{array}{c}37.5 \\
(32-47)\end{array}$ & \\
\hline SOC score, median (IQR) & $\begin{array}{c}63 \\
(56-71)\end{array}$ & $\begin{array}{c}62.5 \\
(55.25-68.75)\end{array}$ & $\mathrm{p}=0.369$ \\
\hline
\end{tabular}

\section{DISCUSSION}

This study is the first to compare SOC between patients with IBD and healthy controls. We found that SOC in IBD patients is similar to SOC of the general population. 
Table II. 13-item SOC median scores in patients with ulcerative colitis (UC), compared with healthy controls and demographic parameters after matching

\begin{tabular}{|c|c|c|c|}
\hline Variable & Controls & $\begin{array}{l}\text { Ulcerative } \\
\text { colitis }\end{array}$ & \\
\hline Women, n (\%) & $41(71.9)$ & $36(63.2)$ & \\
\hline $\begin{array}{l}\text { Education }>12 \text { years, } \\
\mathrm{n}(\%)\end{array}$ & $40(70.2)$ & $41(71.9)$ & \\
\hline \multicolumn{4}{|l|}{ Marital Status, n (\%) } \\
\hline Single & $12(21.1)$ & $13(22.8)$ & \\
\hline In a relationship & $40(70.2)$ & $40(70.2)$ & \\
\hline Past relationship & $5(8.8)$ & $4(7)$ & \\
\hline Employed, n (\%) & $47(82.5)$ & $46(80.7)$ & \\
\hline Age median (IQR) & $\begin{array}{c}35 \\
(32.5-47)\end{array}$ & $\begin{array}{c}39.02 \\
(29.68-46.38)\end{array}$ & \\
\hline $\begin{array}{l}\text { SOC score median } \\
\text { (IQR) }\end{array}$ & $62(55-69)$ & $63.82(56-73)$ & $\mathrm{p}=0.354$ \\
\hline
\end{tabular}

These results are in contrast to those documented among patients with several multifactorial chronic diseases $[13,14,18$ 22]. An observational study that compared SOC levels between patients with diabetes mellitus and healthy controls [13] found that the control population had 2.4 times higher odds of having a high SOC score as compared to patients with type 2 diabetes. On the other hand, reports on the correlation between SOC and disease control had conflicting results. Two studies [19, 20] showed that SOC did not correlate with metabolic control among patients with insulin-dependent diabetes. Another study indicated that higher SOC scores were correlated with a better glycemic control in diabetes type 1 and 2 patients [21].

A study investigating the role of SOC in the development of SLE in women found that women with SLE had significantly lower SOC scores compared with healthy controls, but no significant correlation with disease activity was detected [14].

The current study did not investigate correlations between SOC score and disease severity. Intuitively, we would expect SOC to be related to disease severity, but the results of the diabetes [19] and SLE [14] studies indicate otherwise. Although these studies compared healthy controls to diabetic [13] or SLE [14] patients, it is important to note that groups were not matched. In the diabetes study, there were significant differences in socio-demographic characteristics of age, level of education and employment status between the two study groups [13]. In the SLE study, a significant difference was found only regarding the employment status, and that was related to the physical effects of the disease [14].

According to Antonovsky's theory [10] of salutogenesis, individuals with lower SOC scores are more prone to develop chronic diseases. Therefore, we would expect patients with IBD to have lower SOC scores than healthy controls.

Surprisingly, in contrast to the findings reported with other chronic diseases, we found that in IBD, SOC scores were similar to those of the healthy controls. This can be explained by meticulously matching patients and controls on five major parameters (age, gender, education, employment status and marital status, with less than $5 \%$ absolute difference between groups considered as acceptable for matching). Furthermore, although the 13-items SOC questionnaire was validated on IBD patients [15], this validation was recently challenged by Lerdar et al. [22] who claimed that for IBD, the 13 items should be reduced to 11 items in order to achieve better matching with the 29-items SOC questionnaire.

This study has some limitations. The study population consisted of ambulatory patients who adhere to clinic visits and thus may represent a population of patients who have better SOC, which may indicate selection bias. The data collection method differed between groups: patients answered a questionnaire during a clinic visit, while the controls answered an internet-based questionnaire. However, since the outcomes were similar in both groups, we believe that this methodological difference did not affect the results. In the study design, we insisted on strict matching, which resulted in a smaller study population. Thus, the statistical power of the study was also decreased. Furthermore, the meticulous matching of the two groups might bring parameters that are independently influenced by SOC, such as employment and marital status, into the equation.

Sense of coherence was already investigated among IBD patients [15] and was found to influence the development of anxiety and depression [15] regardless of disease severity. On the other hand, in our recently published study [23] of adherence in IBD patients, multivariate analysis did not find a significant difference in the SOC scores between adherent and non-adherent patients. These results, in addition to those of the current study, raise several questions. The first is whether the salutogenesis theory applies to IBD patients. The second question is whether the SOC questionnaire should be revised for IBD patients, as claimed by Lerdar et al. [22].

The relation between SOC and physical health is more complex and seems to be weaker than the relation between SOC and mental health [16].

\section{CONCLUSION}

The question of whether SOC is associated with the development of chronic disease and particularly with IBD remains open.

Conflicts of interest: None to declare. Financial support: No specific funding was received for this study.

Authors' contributions: A.E.A., T.N., N.R., E.B. performed the research and analyzed the data. A.E.A., T.N., N.R., E.B., A.B.S., Y.M., H.S., S.M., I.L., F.S.B. collected the data. T.N., N.R., E.B. designed the research study. A.E.A., N.R., T.N., E.B. wrote the paper. A.E.A., H.S., S.M., F.S.B., I.L., Y.M., A.S. contributed to the study design. T.Z.B. performed the statistical analyses. All authors had full access to all the data in the study, approved the final draft submitted and the decision to submit it for publication.

\section{REFERENCES}

1. Xavier RJ, Podolsky DK. Unravelling the pathogenesis of inflammatory bowel disease. Nature 2007;448:427-434. doi:10.1038/nature06005

2. Moons P, Norekvål TM. Is sense of coherence a pathway for improving the quality of life of patients who grow up with chronic diseases? 
A hypothesis. Eur J Cardiovasc Nurs 2006;5:16-20. doi:10.1016/j. ejcnurse.2005.10.009

3. Eriksson AK, Ekbom A, Granath F, Hilding A, Efendic S, Ostenson CG. Psychological distress and risk of pre-diabetes and Type 2 diabetes in a prospective study of Swedish middle-aged men and women. Diabet Med 2008;25:834-842. doi:10.1111/j.1464-5491.2008.02463.x

4. Gonzalez JS, Delahanty LM, Safren SA, Meigs JB, Grant RW. Differentiating symptoms of depression from diabetes-specific distress: relationships with self-care in type 2 diabetes. Diabetologia 2008;51:1822-1825. doi:10.1007/s00125-008-1113-x

5. Andreoulakis E, Hyphantis T, Kandylis D, Iacovides A. Depression in diabetes mellitus: a comprehensive review. Hippokratia 2012;16:205214.

6. Goodhand JR, Wahed M, Mawdsley JE, Farmer AD, Aziz Q, Rampton DS. Mood disorders in inflammatory bowel disease: Relation to diagnosis, disease activity, perceived stress, and other factors. Inflamm Bowel Dis 2012;18:2301-2309. doi:10.1002/ibd.22916

7. Häuser W, Janke KH, Klump B, Hinz A. Anxiety and depression in patients with inflammatory bowel disease: comparisons with chronic liver disease patients and the general population. Inflamm Bowel Dis 2011;17:621-632. doi:10.1002/ibd.21346

8. Lin EH, Katon W, Von Korff M, et al. Relationship of depression and diabetes self-care, medication adherence, and preventive care. Diabetes Care 2004;27:2154-2160. doi:10.2337/diacare.27.9.2154

9. Antonovsky A. Sense of Coherence - Orientation to Life Questionnaire. Unraveling the Mystery of Health: How people Manag Stress and Stay well. Jossey-Bass, San Francisco1987:6-8.

10. Antonovsky A. The structure and properties of the sense of coherence scale. Soc Sci Med 1993;36:725-733. doi:10.1016/02779536(93)90033-Z

11. Geyer S. Some conceptual considerations on the sense of coherence. Soc Sci Med 1997;44:1771-1779.doi:10.1016/S0277-9536(96)00286-9

12. Super S, Verschuren WM, Zantinge EM, Wagemakers MA, Picavet HS. A weak sense of coherence is associated with a higher mortality risk. J Epidemiol Community Health 2014;68:411-417. doi:10.1136/ jech-2013-203085
1 3. Merakou K, Koutsouri A, Antoniadou E, et al. Sense of coherence in people with and without type 2 diabetes mellitus: an observational study from Greece. Ment Health Fam Med 2013;10:3-13.

14. Abu-Shakra M, Keren A, Livshitz I, et al. Sense of coherence and its impact on quality of life of patients with systemic lupus erythematosus. Lupus 2006;15:32-37. doi:10.1191/0961203306lu2255oa

15. Freitas TH, Andreoulakis E, Alves GS, et al. Associations of sense of coherence with psychological distress and quality of life in inflammatory bowel disease. World J Gastroenterol 2015;21:6713-6727. doi:10.3748/ wjg.v21.i21.6713

16. Eriksson M, Lindström B. Antonovsky's sense of coherence scale and the relation with health: A systematic review. J Epidemiol Community Health 2006;60:376-381. doi:10.1136/jech.2005.041616

17. Opheim R, Fagermoen MS, Jelsness-Jorgensen LP, Bernklev T, Moum B. Sense of coherence in patients with inflammatory bowel disease. Gastroenterol Res Pract 2014;2014:989038. doi:10.1155/2014/989038

18. Goulia P, Voulgari PV, Tsifetaki N, et al. Sense of coherence and self-sacrificing defense style as predictors of psychological distress and quality of life in rheumatoid arthritis: a 5-year prospective study. Rheumatol Int 2015;35:691-700. doi:10.1007/s00296-014-3134-8

19. Lundman B, Norberg A. The significance of a sense of coherence for subjective health in persons with insulin-dependent diabetes. J Adv Nurs 1993;18:381-386. doi:10.1046/j.1365-2648.1993.18030381.x

20. Richardson A, Adner N, Nordström G. Persons with insulindependent diabetes mellitus: Acceptance and coping ability. J Adv Nurs 2001;33:758-763. doi:10.1046/j.1365-2648.2001.01717.x

21. Cohen M, Kanter Y. Relation between sense of coherence and glycemic control in type 1 and type 2 diabetes. Behav Med 2004;29:175-183. doi:10.3200/BMED.29.4.175-185

22. Lerdal A, Opheim R, Gay CL, Moum B, Fagermoen MS, Kottorp A. Psychometric limitations of the 13-item Sense of Coherence Scale assessed by Rasch analysis. BMC Psychol. 2017;5:18. doi:10.1186/ s40359-017-0187-y

23. Eindor-Abarbanel A, Naftali T, Ruhimovich N, et al. Revealing the Puzzle of Nonadherence in IBD - Assembling the Pieces. Inflamm Bowel Dis 2018;24:1352-1360. doi:10.1093/ibd/izy013 